\title{
Registries reveal real-world use of anticoagulant drugs in AF
}

\section{ce \\ we believe that the results reflect a positive trend in clinical management of patients with $\mathrm{AF}$

F

Data from two registries reveal the 'real-world' use of anticoagulant drugs for stroke prevention in patients with nonvalvular atrial fibrillation (AF). Findings from phase 2 of the international GLORIA-AF registry indicate that non-vitamin $\mathrm{K}$ antagonist oral anticoagulants (NOACs) have been highly adopted in clinical practice, and are now more commonly prescribed than vitamin $\mathrm{K}$ antagonists (VKAs) to patients with newly diagnosed AF in Europe and North America. In a separate propensity-weighted cohort study performed using three national databases in Denmark, the efficacy and safety of low-dose NOACs were generally similar to that of warfarin, but some differences were noted in patients with a clinical indication for dose reduction (such as old age or reduced kidney function).

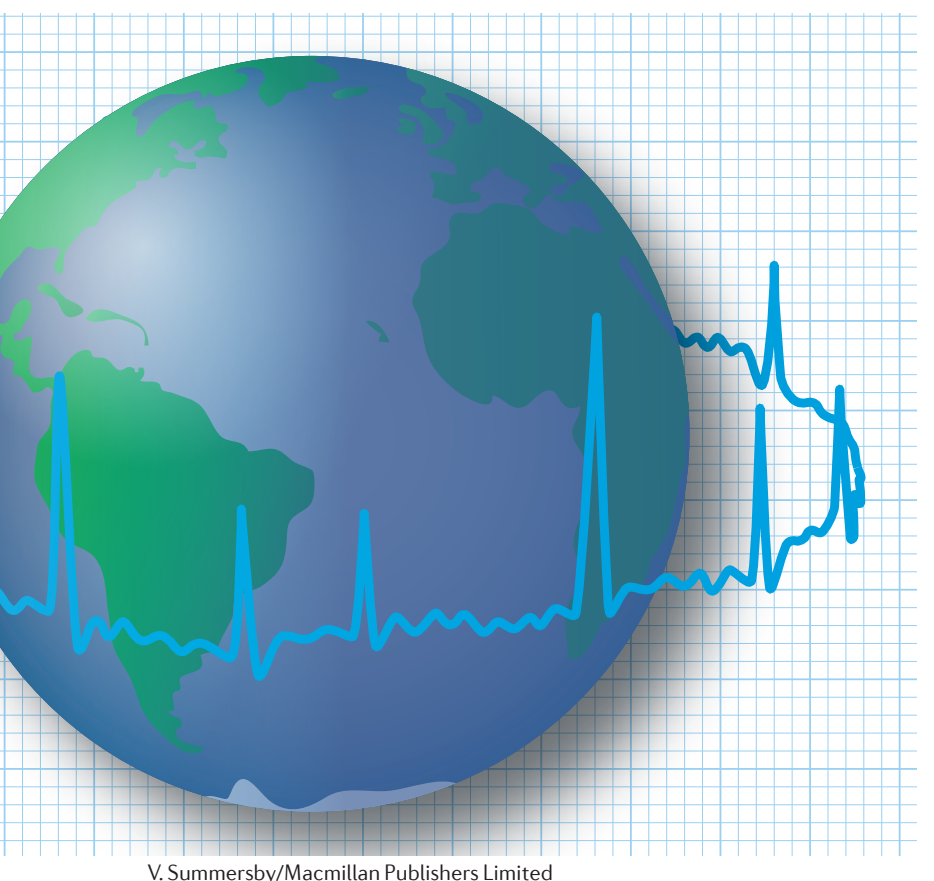

GLORIA-AF is a global, prospective registry documenting antithrombotic treatment in patients with newly diagnosed AF. Phase 2 began with the introduction of dabigatran, the first NOAC to be approved for clinical use. During phase 2, 15,641 patients from 44 countries were enrolled, 15,092 of whom were eligible for analysis. Overall, 79.9\% received oral anticoagulants $(47.6 \%$ NOAC, 32.3\% VKA), $12.1 \%$ received antiplatelet agents, and $7.8 \%$ received no antithrombotic treatment.

Regional variations during phase 2 existed. In Europe $(n=7,108)$, treatment with a NOAC (52.3\%) was more common than with a VKA (37.8\%); $6.0 \%$ of patients received antiplatelet therapy, and $3.8 \%$ received no antithrombotic treatment. In North America (Canada and the USA; $n=3,403)$, treatment with a NOAC (52.1\%) was again more common than with a VKA (26.2\%); $14.0 \%$ received antiplatelet therapy, and $7.5 \%$ received no antithrombotic treatment. By contrast, in Asia $(n=3,071)$, use of NOACs $(27.7 \%)$ was similar to the use of VKAs (27.5\%), with $25.0 \%$ receiving antiplatelet drugs, and $19.8 \%$ receiving no antithrombotic treatment.

A large proportion of patients remain untreated, particularly in Asia and North America. "While this proportion of undertreated patients should not be overlooked, overall we believe that the results reflect a positive trend in clinical management of patients with AF through the relatively rapid adoption of the newer drugs," says Menno Huisman, lead author of the report in J. Am. Coll. Cardiol. "In the ongoing phase 3 of
GLORIA-AF," continues Huisman, "longer-term, 3-year follow-up data will be collected from all patients, irrespective of treatment prescribed, allowing safety and effectiveness analysis for other oral anticoagulants also in a global, prospective cohort."

The separate Danish study included 55,644 patients with AF. Peter Brønnum Nielsen and colleagues had observed that "the proportion of patients in Denmark on [a] reduced-dose regimen was substantially higher than initially anticipated and very different from what was seen in the phase III clinical trials". Overall, 4,400 received apixaban $2.5 \mathrm{mg}$ twice daily, 8,875 received dabigatran $110 \mathrm{mg}$ twice daily, 3,476 received rivaroxaban $15 \mathrm{mg}$ once daily, and 38,893 received warfarin. In the propensity-weighted analyses, no significant differences in efficacy were observed, although ischaemic stroke and systemic embolism tended to be higher with apixaban than with warfarin, and thromboembolism tended to be lower with rivaroxaban or dabigatran than with warfarin. Rates of bleeding were significantly lower with dabigatran, but not significantly different with apixaban or rivaroxaban, compared with warfarin.

Gregory B. Lim

ORIGINAL ARTICLES Huisman, M. V. et al. The changing landscape for stroke prevention in AF: findings from the GLORIA-AF registry phase 2.J. Am. Coll. Cardiol. 69, 777-785 (2017)| Brønnum Nielsen, P. et al. Effectiveness and safety of reduced dose non-vitamin $\mathrm{K}$ antagonist oral anticoagulants and warfarin in patients with atrial fibrillation: propensity weighted nationwide cohort study. BMJ 365, j510 (2017) FURTHER READING Calenda, B. W. et al. Stroke risk assessment in atrial fibrillation: risk factors and markers of atrial myopathy. Nat. Rev. Cardiol. 13 , 549-559 (2016) 